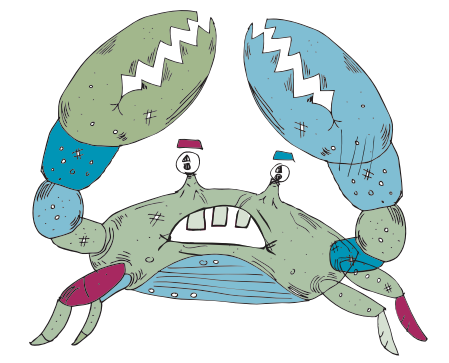

( ANTIMICROBIALS
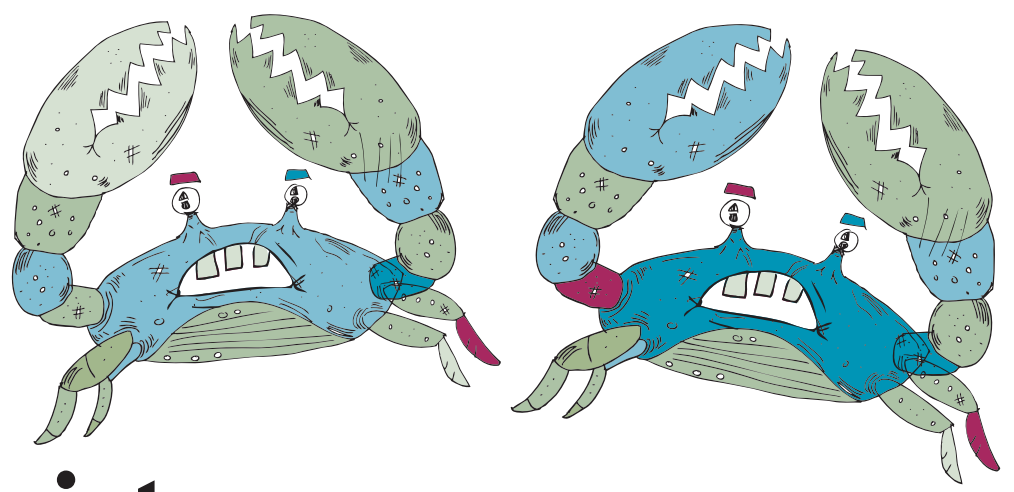

\title{
Reactive resistance
}

Treatment of bacteria with low concentrations of bactericidal antibiotics can generate multidrug resistance through an increase in the mutation rate that is driven by the formation of reactive oxygen species (ROS), according to a report from Mike Kohanski, Mark DePristo and Jim Collins in a recent issue of Molecular Cell.

Previous work from Kohanski, Collins and co-workers had shown that all major classes of bactericidal antibiotics kill cells by inducing the formation of highly toxic hydroxyl radicals, which can damage DNA, proteins and lipids. In this follow-up study, they were interested in investigating whether this process had a mutagenic component. They began by looking at the effects of treating Escherichia coli str. K-12 substr. MG1655 with low concentrations of ampicillin, kanamycin and norfloxacin. All three treatment regimes led to a significant increase in the mutation rate (up to eightfold) compared with untreated controls. Analysis of the oxidative stress levels in treated and untreated cells using a fluorescent reporter dye revealed a correlation between the increased rate of mutation and the generation of ROS. The involvement of ROS was further confirmed by the fact that the addition of thiourea, a hydroxyl radical scavenger, reduced the mutation rates to the levels seen in untreated cells.

The authors speculated that the increased mutagenesis that occurs in response to antibiotic-induced oxidative stress might be involved in the emergence of resistance. Consistent with this idea, they found that treatment with low concentrations of bactericidal antibiotics led to the retention of sensitivity to the test antibiotic but increased the minimum inhibitory concentrations for a range of other antibiotics. Importantly, the authors were able to demonstrate that this phenomenon also occurred in the Gram-positive bacterium Staphylococcus aureus and in a clinical E. coli isolate.

So, bactericidal antibiotics can function as active mutagens and may have a role in the emergence of multidrug resistance. These results have important implications for antibiotic use, in particular for combination therapies.

Sheilagh Molloy

ORIGINAL RESEARCH PAPER Kohanski, M. A. DePristo, M. A. \& Collins, J. J. Sublethal antibiotic treatment leads to multidrug resistance via radical-induced mutagenesis. Mol. Cell 37 , 311-320 (2010) 